\title{
Higgs for graviton: simple and elegant solution
}

\author{
Ali H. Chamseddine ${ }^{a, b}$ and Viatcheslav Mukhanov ${ }^{c, d}$ \\ ${ }^{a}$ Physics Department, American University of Beirut, \\ Beirut, Lebanon \\ ${ }^{b}$ I.H.E.S., \\ F-91440 Bures-sur-Yvette, France \\ ${ }^{c}$ Theoretical Physics, Ludwig Maxmillians University, \\ Theresienstr. 3\%, 80333 Munich, Germany \\ ${ }^{d}$ Department of Physics, New York University, \\ 4 Washington Place, New York, NY 10003, U.S.A. \\ E-mail: chams@aub.edu.1b, Viatcheslav.Mukhanov@physik.uni-muenchen.de
}

ABSTRACT: A Higgs mechanism for gravity is presented, where four scalars with global Lorentz symmetry are employed. We show that in the "broken symmetry phase" a graviton absorbs all scalars and become massive spin 2 particle with five degrees of freedom. The resulting theory is unitary and free of ghosts.

Keywords: Spontaneous Symmetry Breaking, Classical Theories of Gravity, Space-Time Symmetries 
Forty years ago van Dam, Veltman [1] and Zakharov [2] pointed out that the propagator for a massive graviton does not have a smooth limit to the massless case. The action used is that of Fierz and Pauli [3] with mass terms breaking general coordinate invariance explicitly. The straightforward conclusion was that the graviton mass must be mathematically strictly zero rather than some extremely small value because in the presence of discontinuity the massless and massive theories would predict different results either for the perihelion shift or deflection of starlight. This apparent paradox was resolved by Vainstein [4] who found that the massive theory contains a new distance scale below which the massive graviton behave like massless particle and it became clear that the graviton could have small nonvanishing mass which still would not contradict experiments. Over the years, further development of this scale were considered and in [5] it was clearly demonstrated how this mechanism works (see, also [6]).

The analysis by Deser and Boulware [7] lead to the conclusion that the massive theory is ill behaved because in addition to the five degrees of freedom of massive graviton there must be an extra scalar degree of freedom, which does not decouple. Work by Isham, Salam and Strathdee [8] examined a theory of bigravity with a direct mixing mass term, where one of the gravitons becomes massive while the other remained massless. This was generalized by Chamseddine, Salam and Strathdee [9], who considered the mixing mass terms generated through the spontaneous breaking of gauge symmetry. (for further developments in bigravity theories in relation with the graviton mass see [10] and references there). There were also attempts to use theories with extra dimensions. Dvali, Gabadadze, and Porrati [11] have invented a model based on five dimensions with an infinite size extra dimension. Their theory when considered around a true background seems to be free of ghosts. This theory is especially interesting because of the claim of uniqueness [12]. Further interesting steps were made in $[13,14]$, where general relativity with an auxiliary non-dynamical extra dimension was considered with the purpose of obtaining effective massive ghost-free gravity.

It was suspected that the failure of obtaining a ghost free consistent theory for a massive graviton in four dimensions with only one metric, is related to the absence of a ghost free Higgs mechanism that would generate the graviton mass. The string inspired theories, considered in $[15,16]$ are not ghost free when considered around trivial background.

In a promising attempt 't Hooft [17] (see also [18, 19]) exploited a collection of four scalar fields whose vacuum expectation value breaks general coordinate invariance to give mass to the graviton. The kinetic energies of the scalar fields were combined together using the Minkowski metric and thus involving a ghost in the unbroken phase. In the broken symmetry phase the model failed to produce the Fierz-Pauli term for the massive graviton, and the ghost state could not be decoupled.

In this letter we give an elegant solution to the problem of making the graviton massive via Higgs mechanism, and show explicitly how all Higgs fields are absorbed. The resulting spectrum in the broken symmetry phase will consist only of a massive graviton, with FierzPauli mass term and, hence, it has five degrees of freedom. In the unbroken phase we have a massless graviton interacting with four scalar fields, which in the linear approximation lack a propagator. The resulting theory is well defined in all different vacua and is ghost free. 
Let us consider four fields $\phi^{A}, A=0,1,2,3$, which are scalars under coordinate transformations and assume that they posses an extra symmetry with respect to "Lorentz transformations" in the field space. These transformations involve index $A$, thus mixing the scalar fields and preserving the metric $\eta_{A B}=\operatorname{diag}(1,-1,-1,-1)$ in the field space. Next, from $\phi^{A}$ we construct the field space tensor

$$
H^{A B}=g^{\mu \nu} \partial_{\mu} \phi^{A} \partial_{\nu} \phi^{B}
$$

symmetric with respect to $A$ and $B$. The scalar field indices $A$ and $B$ will always be raised and lowered with Minkowski metric $\eta_{A B}$. It is convenient to decompose $H_{B}^{A}$ into trace and traceless parts as

$$
H_{B}^{A}=\tilde{H}_{B}^{A}+\frac{1}{4} \delta_{B}^{A} H
$$

where $H=H_{A}^{A}$ and $\tilde{H}_{A}^{A}=0$.

To demonstrate the idea we will first consider the following action which is explicitly diffeomorphism and Lorentz invariant and provides us the graviton mass term:

$$
S=-\frac{1}{2} \int d^{4} x \sqrt{-g} R+\frac{m^{2}}{2} \int d^{4} x \sqrt{-g}\left[3\left(\left(\frac{1}{4} H\right)^{2}-1\right)^{2}-\tilde{H}_{B}^{A} \tilde{H}_{A}^{B}\right],
$$

where $8 \pi G=1$. It is easy to see that the equations of motion for the metric $g_{\mu \nu}$ and fields $\phi^{A}$ admit the following vacuum Minkowski solution

$$
\left\langle g_{\mu \nu}\right\rangle=\eta_{\mu \nu}, \quad \phi^{A}=x^{A} .
$$

It is this solution that identifies the global Minkowski metric $\eta_{A B}$ with that of space-time $\eta_{\mu \nu}$. We now expand the fields around this vacuum

$$
\phi^{A}=x^{A}+\chi^{A}, \quad g^{\mu \nu}=\eta^{\mu \nu}+h^{\mu \nu},
$$

Introducing

$$
\begin{aligned}
\bar{h}^{A B} & =H^{A B}-\eta^{A B} \\
& =h^{A B}+\partial^{A} \chi^{B}+\partial^{B} \chi^{A}+\eta^{C D} \partial_{C} \chi^{A} \partial_{D} \chi^{B}+h^{A C} \partial_{C} \chi^{B}+h^{B C} \partial_{C} \chi^{A}+h^{C D} \partial_{C} \chi^{A} \partial_{D} \chi^{B},
\end{aligned}
$$

where $h^{A B}=h^{\mu \nu} \delta_{\mu}^{A} \delta_{\nu}^{B}$ and $\partial^{A}=\delta_{\mu}^{A} \eta^{\mu \nu} \partial_{\nu}$, we can rewrite action (3) in the following form

$$
S=-\frac{1}{2} \int d^{4} x \sqrt{-g} R+\frac{m^{2}}{2} \int d^{4} x \sqrt{-g}\left[\left(\bar{h}^{2}-\bar{h}_{B}^{A} \bar{h}_{A}^{B}\right)+\frac{3}{4^{2}} \bar{h}^{3}+\frac{3}{4^{4}} \bar{h}^{4}\right],
$$

where $\bar{h}_{B}^{A}=\eta_{B C} h^{A C}, \bar{h}=\bar{h}_{A}^{A}$. Note that this result is exact and we did not use any approximation to derive it. Moreover the variable $\bar{h}_{B}^{A}$ is diffeomorphism invariant up to an arbitrary order in perturbations.

Now let us consider small perturbations around background (4). Then up to the linear order in perturbations $\chi^{A}$ and $h^{\mu \nu}$,

$$
\bar{h}_{B}^{A}=h_{B}^{A}+\partial_{B} \chi^{A}+\partial^{A} \chi_{B},
$$


Einstein action is invariant under infinitesimal transformations $\tilde{x}=x+\xi$, where metric perturbations around Minkowski space-time transform in a way similar to (8), with $\chi$ replaced by $\xi$. Therefore the full action, up to second order terms could be expressed in terms of $\bar{h}_{B}^{A}$ :

$$
S=\frac{1}{2} \int d^{4} x\left[\bar{h}_{B}^{A, C} \bar{h}_{A, C}^{B}-2 \bar{h}_{C}^{A, C} \bar{h}_{A, D}^{D}+2 \bar{h}_{C}^{A, C} \bar{h}_{, A}-\bar{h}_{, A} \bar{h}^{, A}-m^{2}\left(\bar{h}_{B}^{A} \bar{h}_{A}^{B}-\bar{h}^{2}\right)\right] .
$$

This clearly shows that the Higgs fields $\phi^{A}$ are completely absorbed to form the massive graviton with five degrees of freedom described by Fierz-Pauli mass term. Because we have avoided to include a term linear in $H$ in action (3) the theory is free of ghosts even around a background with $H_{B}^{A}=0$.

One can wonder how four degrees of freedom for the scalar fields (expected naively) could disappear giving only three extra degrees of freedom to the graviton. To understand this let us take the limit of vanishing gravitational constant. In this case we must set $h_{B}^{A}=0$ in equation (8), which then becomes

$$
\bar{h}_{B}^{A}=\partial_{B} \chi^{A}+\partial^{A} \chi_{B}
$$

and in the action

$$
\frac{1}{2} \int d^{4} x\left(\bar{h}^{2}-\bar{h}_{B}^{A} \bar{h}_{A}^{B}\right)=\int d^{4} x\left[\left(\partial_{A} \chi^{A}\right)^{2}-\left(\partial_{A} \chi^{B}\right)\left(\partial^{A} \chi_{B}\right)\right],
$$

one can immediately recognize Maxwell action for " 4 -vector potential" $\chi^{A}$. Thus, around background (4) the perturbations of four scalar fields would lose one degree of freedom, the $\chi^{0}$, to leave three independent physical degrees of freedom. This accidential symmetry is enough to garantee the absence of the propagator for an extra degree when we build the perturbation theory around Minkowski background. In fact, it is clear that the higher order terms being considered only as perturbations cannot induce a propagator for $\chi^{0}$.

The action used is not the most general one. In fact, there exist infinitely many actions which could serve the same purpose. This is not surprising because even in the standard electroweak theory the uniqueness of the Higgs potential is entirely due to the requirement of renormalizability of the theory. In grand unified theories there are many possible choices for the Higgs potential. Our action (3) possess shift symmetry $\phi^{A} \rightarrow \phi^{A}+c^{A}$ where $c^{A}$ are constants, and extra discrete symmetry $H_{B}^{A} \rightarrow-H_{B}^{A}$. However, even these symmetries, which could protect against the appearance of unwanted quantum corrections, are not enough to fix the action unambiguously.

At first glance a possible simple action which could serve the purpose is

$$
\frac{m^{2}}{2} \int d^{4} x \sqrt{-g}\left(\bar{h}^{2}-\bar{h}_{B}^{A} \bar{h}_{A}^{B}\right)
$$

which, when rewritten in terms of $H_{B}^{A}=\delta_{B}^{A}+\bar{h}_{B}^{A}$, takes the form

$$
\frac{m^{2}}{2} \int d^{4} x \sqrt{-g}\left(H^{2}-H_{B}^{A} H_{A}^{B}-6 H+12\right) .
$$


In this form it is clear that the linear term contains a ghost. Nevertheless this problem can be easily fixed by adding to the action terms which are not "Lorentz invariant" with respect to transformations in the space of field configurations. There is nothing wrong with such terms because these still preserve diffeomorphism and space-time Lorentz invariance. The only thing about which we have to take care of is that the corresponding terms will not spoil the action in quadratic order around Minkowski background (4). For example, if we add to $(12)$ the term $2\left(H_{0}^{0}-1\right)^{3}$ the ghost disappears and around Minkowski background the action (12) is modified to

$$
\frac{m^{2}}{2} \int d^{4} x \sqrt{-g}\left(\bar{h}^{2}-\bar{h}_{B}^{A} \bar{h}_{A}^{B}+2\left(\bar{h}_{0}^{0}\right)^{3}\right) .
$$

The last term here looks like a Lorentz violating term. However as we have stressed above this does not mean that we have abandoned the fundamental Lorentz invariance of spacetime. Note that around a trivial background with $H_{B}^{A}=0$ the linearized scalar fields are propagating and have three degree of freedom.

Returning back to action (3) we find that the trace of the energy momentum of the scalar fields is equal to

$$
T_{\mu}^{\mu}=\frac{m^{2}}{2}\left(\frac{3}{128} H^{4}-6\right),
$$

and therefore energy is bounded from below. This action is ghost free in linear order around both, trivial and Minkowski, backgrounds. On the other hand, because the time derivative of the fields appear in the action in the combination

$$
\left(\dot{\phi}^{0}\right)^{2}-\left(\dot{\phi}^{i}\right)^{2}
$$

one may worry that the phase space of $\left(\dot{\phi}^{0}\right)^{2}$ might be unbounded and the problem with ghosts can reappear at the nonlinear level. This problem can be easily solved by adding to the action terms which depend only on $H_{0}^{0}$ and do not modify the action at quadratic order around Minkowski background.

When the background scalar fields disappear, that is $H_{B}^{A}=0$, the graviton decouples from the scalar fields and becomes massless. In this case, however, there appears negative cosmological constant of order $m^{2}$ and the solution of the Einstein equations is anti de Sitter space. One can naturally ask whether the appearance of a negative cosmological constant is an inherent property needed for producing the graviton mass via Higgs mechanism? In fact, it is not the case and we can easily find an action with zero or positive cosmological constant. For example, let us consider

$$
\frac{m^{2}}{2} \int d^{4} x \sqrt{-g}\left[\left(\left(\frac{1}{4} H\right)^{2}-1\right)^{2}\left(\alpha\left(\frac{1}{4} H\right)^{2}-\beta\right)-\tilde{H}_{B}^{A} \tilde{H}_{A}^{B}\right] .
$$

If the constants $\alpha$ and $\beta$ satisfy the condition $\alpha-\beta=3$ then this action provides the FierzPauli term in the broken symmetry state. In the unbroken phase with $H_{B}^{A}=0$ the above action reduces to the action with only a cosmological constant $\Lambda=-\frac{1}{2} m^{2} \beta$. Thus, taking 
$\alpha=3$ and $\beta=0$ we obtain that the cosmological constant is zero in broken as well as in unbroken phase and hence Minkowski space-time is the solution of Einstein equations in both cases. Another interesting choice of parameters is $\alpha=2$ and $\beta=-1$, corresponding to a positive cosmological constant of order $m^{2}$ in the unbroken phase. In this case, either the graviton has mass $m$ in broken symmetry phase, or has a vanishing mass (in unbroken phase) with a cosmological constant of order $m^{2}$. Let us take $m \sim H_{0}$, where $H_{0}$ is the value of the Hubble constant today. Then we obtain that the theory under consideration inevitably leads either to modification of gravity on Vainstein scale, which is $H_{0}^{-1}$, or to the presence of a cosmological constant of order $H_{0}^{2}$. This opens an interesting possibility for interpretation of dark energy in the universe.

Finally we would like to know what is happening in the limit $m^{2} \rightarrow 0$. Let us take for definiteness action (3). Redefining the fields, $H_{B}^{A} \rightarrow \hat{H}_{B}^{A}=\sqrt{m} H_{B}^{A}$, and taking the limit $m^{2} \rightarrow 0$, then action (3) reduces to

$$
-\frac{1}{2} \int d^{4} x \sqrt{-g} R+\frac{3}{2} \int d^{4} x \sqrt{-g}\left(\frac{1}{4} \hat{H}\right)^{4} .
$$

The broken symmetry phase with $\hat{H}=4$ corresponds to a huge negative cosmological constant of the order of Planck value. Therefore it is clear that the only solution is Minkowski space with $\hat{H}=0$ and massless graviton.

\section{Acknowledgments}

We are grateful to C. Deffayet, G. Dvali, G. Gabadadze, J. Garriga and W. Siegel for helpful comments and discussions. The work of AHC is supported in part by the National Science Foundation grant 0854779. V.M. is supported by TRR 33 "The Dark Universe" and the Cluster of Excellence EXC 153 "Origin and Structure of the Universe".

Open Access. This article is distributed under the terms of the Creative Commons Attribution Noncommercial License which permits any noncommercial use, distribution, and reproduction in any medium, provided the original author(s) and source are credited.

\section{References}

[1] H. van Dam and M. J. G. Veltman, Massive and mass-less Yang-Mills and gravitational fields, Nucl. Phys. B 22 (1970) 397 [SPIRES].

[2] V.I. Zakharov, Linearized gravitation theory and the graviton mass, JETP Lett. 12 (1970) 312 [Pisma Zh. Eksp. Teor. Fiz. 12 (1970) 447] [SPIRES].

[3] M. Fierz, W. Pauli, On relativistic wave equations for particles of arbitrary spin in an electromagnetic field, Proc. Roy. Soc. Lond. A 173 (1939) 211 [SPIRES].

[4] A.I. Vainshtein, To the problem of nonvanishing gravitation mass, Phys. Lett. B 39 (1972) 393 [SPIRES].

[5] C. Deffayet, G. R. Dvali, G. Gabadadze and A. I. Vainshtein, Nonperturbative continuity in graviton mass versus perturbative discontinuity, Phys. Rev. D 65 (2002) 044026 [SPIRES]. 
[6] E. Babichev, C. Deffayet, R. Ziour, Recovering General Relativity from massive gravity, Phys. Rev. Lett. (2009) 103 [SPIRES].

[7] D. G. Boulware and S. Deser, Can gravitation have a finite range?, Phys. Rev. D 6 (1972) 3368 [SPIRES].

[8] C. J. Isham, A. Salam and J. A. Strathdee, F-dominance of gravity, Phys. Rev. D 3 (1971) 867 [SPIRES].

[9] A.H. Chamseddine, A. Salam and J.A. Strathdee, Strong Gravity and Supersymmetry, Nucl. Phys. B 136 (1978) 248 [SPIRES].

[10] N. Arkani-Hamed, H. Georgi and M.D. Schwartz, Effective field theory for massive gravitons and gravity in theory space, Ann. Phys. 305 (2003) 96 [hep-th/0210184] [SPIRES].

[11] G. R. Dvali, G. Gabadadze and M. Porrati, $4 D$ gravity on a brane in 5D Minkowski space, Phys. Lett. B 485 (2000) 208 [SPIRES]

[12] G. Dvali, S. Hofmann and J. Khoury, Degravitation of the cosmological constant and graviton width, Phys. Rev. D 76 (2007) 084006 [hep-th/0703027] [SPIRES].

[13] G. Gabadadze, General Relativity With An Auxiliary Dimension, Phys. Lett. B 681 (2009) 89 [arXiv:0908.1112] [SPIRES].

[14] C. de Rham, Massive gravity from Dirichlet boundary conditions, Phys. Lett. B 688 (2010) 137 [arXiv:0910.5474] [SPIRES].

[15] M.B. Green and C.B. Thorn, Continuing between closed and open strings, Nucl. Phys. B 367 (1991) 462 [SPIRES].

[16] W. Siegel, Hidden gravity in open string field theory, Phys. Rev. D 49 (1994) 4144 [hep-th/9312117] [SPIRES].

[17] G. 't Hooft, Unitarity in the Brout-Englert-Higgs Mechanism for Gravity, arXiv:0708.3184 [SPIRES].

[18] Z. Kakushadze, Massless Limit of Gravitational Higgs Mechanism, Int. J. Geom. Meth. Mod. Phys. 05 (2008) 157 [arXiv:0711.0386] [SPIRES].

[19] D.A. Demir and N.K. Pak, General Tensor Lagrangians from Gravitational Higgs Mechanism, Class. Quant. Grav. 26 (2009) 105018 [arXiv: 0904.0089] [SPIRES]. 